\title{
EL REALCE: UNA PROPUESTA PARA SU CONCEPTUALIZACIÓN E IDENTIFICACIÓN
}

\author{
Adrián Vergara Heidke
}

\begin{abstract}
RESUMEN
Los elementos destacados en los textos se han abordado desde distintas perspectivas teóricas dentro de la lingüística. En este artículo, luego de hacer una revisión crítica de diferentes conceptos que intentan aprehender este fenómeno, se propone una nueva forma de conceptualizar y analizar (identificar) el realce. Esto se efectúa desde una perspectiva que considera que: en primer lugar, los emisores no son conscientes de todos los elementos lingüísticos y semióticos que enuncian; en segundo lugar, no todo recurso lingüístico y semiótico se asocia con un(os) efecto(s) particular(es); y, en tercer lugar, los conceptos lingüísticos textuales deben poderse aplicar en textos multimodales y no exclusivamente en lo verbal. Finalmente, se señala la necesidad de profundizar en el estudio del realce para delimitar mejor el concepto y hacerlo más práctico.

Palabras claves: realce, intensificación, foco, énfasis, multimodalidad.
\end{abstract}

\begin{abstract}
The elements highlighted in the texts have been approached from different theoretical perspectives within linguistics. In this article, after a critical review of different concepts that attempt to apprehend this phenomenon, we propose a new way to conceptualize and analyze (identify) the emphasis (realce). This is done from a perspective that considers: first, issuers are not conscious of all the linguistic and semiotic elements enunciated, secondly, not all linguistic and semiotic resource is associated with an individual(s) effect(s), and, thirdly, textual linguistic concepts should be applicable in multimodal texts and not exclusively on the verbal dimension. Finally, it emphasizes the need for further study to better delineate enhancement concept and make it more practical.

Key words: enhancement, intensification, emphasis, salience, multimodality
\end{abstract}

\section{Introducción}

En muchas ocasiones, dentro de los textos se encuentran elementos o grupos de elementos ${ }^{1}$ que sobresalen más que otros, los cuales pueden o deben (desde el punto de vista del emisor) llamar la atención de los perceptores (lectores, oyentes, televidentes) y cumplir

Dr. Adrián Vergara Heidke. Profesor de la Escuela de Filología, Lingüística y Literatura, Departamento de Lingüística. Universidad de Costa Rica.

Correo electrónico: adrian.vergara@ucr.ac.cr

Recepción: 27- 07- 2012

Aceptación: 08- 10- 2012 
distintas funciones, por ejemplo, resaltar la actitud (emociones) del emisor o algún contenido semántico. Así, en Bolocco ${ }^{2}$ pide pisco “original” en Perú ${ }^{3}$ se observa que mediante un recurso gráfico ("”) se resalta la palabra original, esto atrae la atención del lector (en particular, chilenos y peruanos) sobre ella y refuerza su significado, además de que se pueden generar en su mente implicaciones, significados asociados ${ }^{4}$ y efectos perlocutivos, debido, por supuesto, a conocimientos culturales previos. Lo anterior se debe al contexto social, en el que Perú y Chile tienen una "disputa" 5 por el origen del pisco. El problema de este fenómeno, que nosotros denominaremos realce, radica en: a) la gran cantidad de recursos que se pueden utilizar para resaltar algún elemento (por ejemplo, recursos gráficos: negritas, cursivas, comillas, signos de exclamación, tamaño, tipografía y color de la letra, dibujos, fotografías, títulos; recursos prosódicos: pausas, entonación, vocalizaciones, alargamientos vocálicos, fuerza de la voz; recursos léxicos: superlativos, intensificadores, palabras con cargas emotivas fuertes; recursos sintácticos: oposiciones, repeticiones, enumeraciones, estructuras sintácticas especiales; recursos sonoros ${ }^{6}$ : silencios, aumentos de volumen), b) la variedad de funciones que cumplen (expresiva, al resaltar la actitud o emoción del hablante; referencial, al resaltar un significado o sentido sobre otro de un término; conativa, al buscar generar una respuesta del receptor; informativa, al destacar un elemento de la estructura informativa), c) los diferentes efectos generados (sensaciones, emociones, mejor almacenaje en la memoria), d) la no correspondencia entre su realización y una finalidad por realzar (por ejemplo, pausas por distracción o duda que resaltan un elemento; realce debido a características del género discursivo o del modelo de texto) y, e) la perspectiva que se adopta para abordarlo (desde el emisor, desde el receptor, desde el texto, desde la interacción). Todo esto dificulta delimitar el fenómeno, es decir, establecer los parámetros para su identificación y definición, ya que los recursos, funciones, efectos, finalidad no le son exclusivos.

En este contexto, en el presente artículo exponemos una propuesta teórica para delimitar e identificar el fenómeno desde un enfoque construccionista y que considera los textos multimodales. En el marco de este objetivo, el artículo comienza con una revisión de cinco propuestas desde diferentes perspectivas lingüísticas. A continuación, se presenta en detalle nuestra propuesta y se cierra con las tareas futuras que se deberían realizar para confirmar, precisar y corregir la propuesta.

\section{Revisión de propuestas teóricas y metodológicas existentes ${ }^{7}$}

La complejidad de este fenómeno ha motivado que, desde distintas perspectivas dentro de la lingüística, se haya intentado abordarlo como un todo o parte de éste. A continuación presentaremos sucintamente algunos acercamientos ${ }^{8}$.

\subsection{Foco}

El concepto de foco proveniene de la gramática funcional y, particularmente, de la estructura informativa de las oraciones, sin embargo, en la teoría lingüística no hay una clara definición de este concepto (Dimroth 2004; Face 2002; Klein 1992; Pinuer 2009). Por ejemplo, en Dimroth (2004) se identifican ${ }^{9}$ tres usos del término como realce, alternativa y lo dicho ${ }^{10}$, mientras que en Alcaraz y Martínez (2004: 269) se presentan dos acepciones de foco: como información nueva en oposición a información dada (distinción tema-rema) y como "unidad sintáctica al que el emisor le atribuye mayor relieve desde el punto de 
vista informativo". Nos detendremos en este último uso del término, por cuanto se acerca al fenómeno que deseamos abordar.

En Gutiérrez (1997: 33-34) se señala que foco se produce cuando el hablante intenta (a partir de una necesidad de hacerlo) llamar la atención sobre algún elemento con el fin de advertir la carga semántica de él, oponiéndolo a algún otro elemento virtual del eje paradigmático, y no alterando la visión representativa de la secuencia. Esta definición presenta algunas dificultades. En primer lugar, el hablante focaliza un elemento a partir de una necesidad y con un fin determinado, por ende, constituye un acto consciente, lo cual requiere trabajar con este presupuesto que carece de una comprobación empírica ${ }^{11}$. En segundo lugar, el fin es advertir la carga semántica del elemento focalizado, lo que deja de lado cualquier carga expresiva de los enunciados. Esto hace caso omiso de los aspectos expresivos, emotivos, subjetivos del uso de la lengua. Si bien en Gutiérrez (1997) se ocupan de lo sintáctico y lo semántico y se excluyen aspectos expresivos y pragmáticos, se introduce al hablante en su definición; por ende, estos aspectos no se deberían ignorar en el análisis y explicación. En tercer lugar, en este texto, el foco tiene una dimensión opositiva paradigmática, es decir, un elemento ha sido resaltado con el fin (en parte) de oponerlo a otro que podría aparecer en su lugar: un elemento in absentia (1997: 33). Destacamos este último punto, sin embargo, consideramos que en algunos casos se presenta esta oposición, pero en los mismos o en otros casos, la oposición (el contraste) se da también en el eje sintagmático, donde el elemento realzado le quita "protagonismo" a los otros elementos del sintagma. Finalmente, en este texto se señala que existen varios recursos lingüísticos para realizar foco (1997: 34), sin embargo, no se establecen los criterios (parámetros o procedimientos) por los cuales se puede determinar que un particular uso constituye un foco. Pareciera, según ese punto de vista, que los únicos recursos para focalizar son los que este autor presenta y que cada vez que se utilizan se está focalizando.

\section{2. Énfasis}

Otros autores recurren al término énfasis para referirse al fenómeno que nos interesa. Así, en Metzeltin ${ }^{12}$ se señala: "A lo largo de la comunicación el emisor puede dar realce a toda una frase o a un término de una frase para llamar sobre ella/él la atención del interlocutor. [...] el término enfatizado corresponde a un centro de interés [...] afectivo y momentáneo que normalmente sólo condiciona la estructuración de la frase en que se encuentra" (1990: 169). De esta definición destacamos que: en primer lugar, el elemento enfatizado puede corresponder a un solo término o a toda una frase (oración), lo cual le otorga mayor alcance al concepto; y, en segundo lugar, sería un centro de interés afectivo, es decir, toma relevancia lo emocional de los interlocutores, aspecto muchas veces dejado de lado en la teoría lingüística. Sin embargo, no compartimos el hecho de presuponer cierto grado de conciencia y una finalidad en el emisor (hablante) previos a la realización del énfasis. Además, no se establecen criterios (parámetros o procedimientos) por los cuales se pueda delimitar este fenómeno.

Asimismo, en Metzeltin se proponen dos tipos de énfasis: uno absoluto y otro contrastivo (1990: 169). El primero se refiere a aquellos recursos que por sí mismos son enfatizadores (algunos adverbios, frases exclamativas, ubicación inesperada de un elemento, entre otros), es decir, que su aparición siempre producirá énfasis. El énfasis contrastivo se da cuando se "[pone] de relieve por contraste explícito o implícito con otros de la misma clase referencial que podrían ocupar la misma casilla sintagmática” (1990: 169). De esta manera, 
se establece una distinción entre recursos de énfasis por sí mismo y el énfasis por contraste en el eje paradigmático (“otros de la misma clase referencial”). Este es un aspecto valioso, ya que propone un criterio de delimitación del fenómeno: siempre que se utilicen los recursos del énfasis absoluto, habrá énfasis ${ }^{13}$. El problema son los parámetros para determinar el énfasis contrastivo.

\subsection{Realce}

En Vigara se utiliza el término 'realce' para denominar un fenómeno lingüístico: "aquel por el que el hablante, obedeciendo a su personal impulso, destaca cara a su interlocutor una parte de su enunciado (que puede ser la acción, una cualidad, un objeto, un sujeto, etc.) o su propia actitud de comunicación" (1992: 131). Además, el realce, en este texto, tendría una "función fundamentalmente expresiva” (1992: 131), por lo menos, en el uso coloquial ${ }^{14}$. Ahora bien, a partir de esta definición cabe preguntarse ¿cuáles son los criterios o procedimientos para determinar si un fenómeno constituye realce?; ¿sólo cuando un hablante destaca conscientemente (con esa meta) una parte de su enunciado corresponde realce?; ¿se diferencia el realce de otros fenómenos como el énfasis o la intensificación?; ¿qué papel juega el interlocutor?; ¿qué efecto tiene el realce o la parte realzada en el todo de un texto?

En Vigara (1992: 77, 131) se señala que el fenómeno que se aborda es de difícil delimitación y que en su análisis se procede por intuición, por lo cual, no se establecen criterios claros -a excepción del presupuesto de un "orden sintáctico no marcado", es decir, no expresivo, que sirve de criterio para el procedimiento llamado "dislocamiento sintáctico" (1992: 73-74)para determinar que algo sea un realce. De esta manera, la intuición de cada investigador se encarga de establecer qué corresponde a un realce ${ }^{15}$. Dejar tan abierto los criterios o procedimientos que delimitan un fenómeno lingüístico conlleva, por lo menos, dos problemas: en primer lugar, la intuición no es un aspecto que se comparta, o sea, todas las personas no poseemos la misma intuición (o intuimos lo mismo), por lo cual esta no constituye un criterio que se pueda generalizar; y, en segundo lugar, lo anterior no permite el diálogo intersubjetivo entre los especialistas y el desarrollo científico, por cuanto no existen los parámetros para replicar el mismo análisis o realizar estudios similares sobre el fenómeno en otros usos de la lengua.

En cuanto al papel del hablante, en este texto se le asigna la responsabilidad de realzar "una parte de su enunciado [...] o su propia actitud de comunicación” (1992: 131), “obedeciendo a un personal impulso" (1992: 131). Esto supone que el hablante es consciente de lo realizado y presenta una predisposición para destacar un elemento. La base de este argumento es la posibilidad de elección que tiene el hablante entre diferentes recursos lingüísticos (1992: 56). Sin embargo, como investigadores no podemos presuponer ese deseo del hablante sin consultarles a los mismos hablantes o sin que el desarrollo del texto ${ }^{16}$ nos permita determinar o inferir esa voluntad. ¿Cómo se puede partir de ese presupuesto en los casos en que los hablantes realizan actos inconscientes, por ejemplo, pausas (con la pausa se podría resaltar algo), ralentización en la oralidad (se puede destacar algo al enunciarlo más lento), repeticiones tanto por inseguridad como por distracción? ¿Constituyen realce estos casos? ¿Cómo podemos decir que el hablante no tuvo la voluntad de realzar sin consultarle o sin seguir el desarrollo de la conversación? En resumen, se necesita de parámetros lingüísticos y, por supuesto, del contexto particular de la interacción ${ }^{17}$ para determinar si se trata de un caso de realce o no.

Por otro lado, en Vigara no se aclara explícitamente la diferencia entre el realce y otros fenómenos similares estudiados en la lingüística. De hecho, pareciera considerarlos como el 
mismo fenómeno: “«Poner de relieve», «reforzar», «enfatizar», «intensificar» [...] y ahora rebautizamos nosotros- realzar. Con todos estos términos se pretende acotar un fenómeno complejo [...]" (1992: 131). ¿Por qué se tuvo la necesidad de rebautizar un fenómeno que ya ha sido abordado con otros términos? Creemos que esa necesidad se da, en primer lugar, para llamar la atención sobre la expresividad del hablante y, en segundo lugar, por intuición, ya que la autora (por lo menos en este texto) se percata que, por ejemplo, el énfasis y la intensificación se refieren a otros fenómenos (corresponderían a dos procedimientos para realzar), sin embargo, como no otorga criterios para delimitar el realce, no deja claras las fronteras entre los fenómenos, lo cual se presta para confusiones ${ }^{18}$.

En esta postura, el rol del interlocutor parece ser secundario. Sólo constituye un elemento del contexto de la interacción, al cual, eso sí, se dirige el realce, pero no desempeña un papel relevante ni como criterio para delimitar el fenómeno ni como lugar de producción de efectos del realce. Asimismo, tampoco se señalan posibles efectos del realce en la interacción, en el desarrollo del texto o en la interpretación. Entonces, nacen preguntas sobre cuál es la relevancia del realce y de su análisis, ¿es sólo un recurso para manifestar la expresividad del hablante?; ¿no interesa cómo lo perciban los receptores?; ¿si se utiliza de manera recurrente en un texto, deja de ser realce?

\subsection{Intensificación}

En Albelda ${ }^{19}$ (2005), por su parte, se aborda el concepto de intensificación. En su trabajo doctoral $^{20}$, esta autora intenta, desde la pragmática, dar una definición del fenómeno que permita establecer criterios claros para su delimitación. Para esto, se propone, en primer lugar, abordar la intensificación -siempre entendiéndola como categoría pragmática- desde tres esferas interdependientes ${ }^{21}$ : la de codificación, la de comunicación y la de relación social (2005: 184). A partir de este análisis se llega a las siguientes propuestas y conclusiones:

\footnotetext{
En definitiva, la intensificación pragmática es una estrategia evaluativa que se encuentra en el contenido proposicional o en la modalidad; el hablante la elige conscientemente con una finalidad: producir determinados efectos a nivel comunicativo y social. Los rasgos que conforman la intensificación, escalaridad, evaluación y ausencia de modificación del contenido proposicional, están al servicio de su valor estratégico: posibilitan que se creen unos compromisos u obligaciones entre los propios interlocutores y/o entre estos y el mensaje. El aumento de estos compromisos se ha venido explicando en la bibliografía como un aumento de grado de la fuerza ilocutiva. [...] La intensificación se manifiesta de forma diferente según si atendemos a los efectos que produce en el nivel monológico o en el dialógico. En el ámbito monológico, los intensificadores son mecanismos con los que se refuerza el punto de vista, la intención del hablante, es decir, se refuerza la fuerza ilocutiva del acto de habla en cuestión. A nivel dialógico, la intensificación de la fuerza ilocutiva influye en la relación con el otro interlocutor. Desde esta perspectiva, los intensificadores son mecanismos de regulación interactiva mediante los que se persigue la negociación del acuerdo o desacuerdo entre los interlocutores. (2005: 286-287)
}

En esta cita se observa uno de los mejores intentos por delimitar, en este caso, no el fenómeno que nos interesa, pero sí una parte de éste. Desde la pragmática se abarca no sólo el código, el lenguaje verbal, sino también los participantes de un acto comunicativo, lo cual se toma en cuenta en la definición y en el análisis. De la propuesta de Albelda (2005) -además de su profundidad y claridad- rescatamos, en primer lugar, que considera la intensificación como una categoría pragmática, por ende, sólo se puede acceder a ella y comprenderla desde esa perspectiva teórica. Con esto la autora deja delimitado el campo de acción del concepto. En segundo lugar, propone rasgos característicos de la intensificación (escalaridad, evaluación y ausencia de modificación del contenido proposicional) a partir de los cuales se la puede 
identificar y analizar, además de marcar explícitamente sus fronteras y, así, diferenciarla de otros fenómenos. En tercer lugar, establece distintos efectos si se toma en cuenta el nivel monológico o el dialógico. De este modo, la autora no se queda en un único nivel, con la consecuente limitación de ese acercamiento, y muestra que el uso de esta estrategia conlleva variaciones en los efectos posibles. Finalmente, se preocupa de los efectos que produciría la intensificación tanto en el mensaje como en la interacción misma, es decir, en el hablante, su interlocutor y la relación entre ellos.

No obstante lo anterior, también hay algunos puntos que consideramos problemáticos de la propuesta de Albelda (2005). Al proponer que el hablante intensifica "conscientemente 22 ", la autora cierra la posibilidad a cualquier enunciado "intensificado" inconscientemente por el hablante. Ante esto cabe preguntarse, ¿qué pasa cuando se cumplen los criterios de la intensificación pero el hablante intensificó inconscientemente?, ¿cómo se lo interpreta y se lo clasifica?, ¿existiría otra categoría para esto? Asimismo, la autora señala explícitamente que los hablantes intensificarían para lograr una determinada finalidad, es decir, ellos tienen completa conciencia de lo que están realizando. En consecuencia, se deja de lado cualquier enunciado (aparentemente) intensificado que no cumpla los requisitos previos de conciencia de la realización y conciencia de la finalidad. Por otro lado, la evaluación, como rasgo característico de la intensificación, presenta algunos aspectos complejos para su abordaje, por cuanto se refiere una valoración subjetiva del hablante. Si bien nos parece acertado el rasgo como criterio delimitador, no se entregan suficientes herramientas para determinar si hubo evaluación y sobre qué valores personales o sociales se estableció esa evaluación, lo cual hace que cada investigador establezca (muchas veces de manera implícita) sus criterios para ese fin. Finalmente, esta autora se acerca a la intensificación desde la pragmática y se concentra en conversaciones coloquiales, por lo que su delimitación del fenómeno o, mejor dicho, algunos de los criterios utilizados son difíciles de generalizar, por ejemplo ¿cómo se aborda la evaluación en entrevistas periodísticas (en las que pueden faltar fragmentos)? O ¿cómo se aborda la finalidad del hablante y los posibles efectos en el interlocutor cuando existe distancia espacial y temporal entre ellos?

\subsection{Hervorhebung}

En el ámbito germano (Klein ${ }^{23} 1992$ Kunze $^{24}$ 1991; trabajos referidos ${ }^{25}$ en Vigara 1992; y en Kiesler 1989) se han acercado al fenómeno que nos interesa y para identificarlo han utilizado el término Hervorhebung ${ }^{26}$. Este término puede traducirse como realce, énfasis, poner de relieve, destacar, resaltar. De los distintos trabajos existentes nos detendremos en el de Reinhard Kiesler (1989), por cuanto propone la utilización de cuatro procedimientos para la identificación del fenómeno, los cuales consideramos de utilidad. Este autor da la siguiente definición:

Realce es cada cambio durante un proceso de producción lingüística [uso de la lengua] -que se refleja en el nivel locucionario-, el cual tiene, de manera consciente o inconsciente y de orientación teleológica, por objetivo la intensificación del efecto perlocucionario deseado del enunciado. Por medio de este enunciado modificado se transmite más información ${ }^{27}$. (1989: 82)

Hervorhebung constituye un proceso contrastivo ${ }^{28}$, ya que se presupone la posibilidad de emitir otro enunciado similar ${ }^{29}$ sin llevar a cabo Hervorhebung, el cual sería la "posibilidad normal" (Normalmöglichkeit) de enunciación.

Kiesler sigue los planteamientos de Austin (1962) sobre los actos de habla, por lo que el nivel locucionario se refiere al acto de decir algo, el cual se compone de tres actos (acto 
fonético, fáctico y rético), y el perlocucionario a los efectos en el oyente, el hablante o una tercera persona que se producen directa o indirectamente a partir de actos locucionarios e ilocucionarios. De esta manera, Hervorhebung se da en el nivel formal (locucionario) de los enunciados, pero con el objetivo de intensificar determinados efectos deseados ${ }^{30}$, todo lo cual conlleva un refuerzo ${ }^{31}$ de la información. Ahora bien, ¿qué sucede en el nivel ilocucionario cuando se da Hervorhebung?, Kiesler señala que es una pregunta todavía abierta (1989: 86). De la definición anterior destacamos el rol fundamental que juegan los efectos (el nivel perlocucionario) en la realización de la Hervorhebung, ya que no sólo se considera el nivel informativo (Gutiérrez 1997) y expresivo (Vigara 1992; Albelda 2005), sino que también lo que se produciría en los interlocutores ${ }^{32}$ (Albelda 2005). Sin embargo, este autor considera que los hablantes tienen consciente o inconscientemente como objetivo producir determinados efectos (actos perlocucionarios), lo cual trae consigo la dificultad que se presupone una predisposición de los hablantes por producir Hervorhebung. Al igual que en los trabajos anteriormente expuestos, se obliga a los investigadores a partir de esa presuposición, o a consultarle a los hablantes si tenían o no la finalidad de realizar Hervorhebung.

Kiesler, además de dar una definición semántica-pragmática ${ }^{33}$, propone cuatro procedimientos para identificar cuándo estamos frente a una Hervorhebung o no. Para esto aplica categorías de la retórica clásica: inserción, deleción, permutación y sustitución (1989: 101-103). Todos estos procedimientos se realizan a partir de la posibilidad normal. Así, inserción es el procedimiento mediante el cual se le introduce un elemento (o grupo de elementos) a la posibilidad normal: "[...] y hoy creo que era bastante así [...]"34 (1989: 101), donde "bastante" se ha insertado a la posibilidad normal, "[...] y hoy creo que era así [...]"35 para producir Hervorhebung. La deleción consiste en eliminación de algún(os) elemento(s): de "[...] y tu burra es un excelente animal. Fue negocio [...]"36 (1989: 102), se construye la posibilidad normal "[...] y tu burra es un excelente animal. Fue un buen [muy buen, óptimo] negocio", de la cual no se enunciaría algún calificativo positivo de 'negocio' ("un buen”, "un muy buen", "óptimo”). El procedimiento de permutación corresponde al cambio de orden dentro del enunciado, lo cual supone un orden "normal" dentro de la lengua: en el ejemplo "De ideologías estamos todos hartos"37 (1989: 102), se habría cambiado el orden normal que sería "Todos estamos hartos de ideologías". En la sustitución se cambia un elemento por otro: en "Serían como las diez cuando llegue a la ciudad"38 (1989: 103), se utilizó "serían como" en lugar de "Eran" de la posibilidad normal "Eran las diez cuando llegué a la ciudad". Si bien algunos de los ejemplos o de las posibilidades normales utilizados por Kiesler (1989) pueden ser discutibles (la deleción, la inserción o la sustitución), consideramos que la propuesta y el uso explícito de estos procedimientos constituyen un aporte para la teoría lingüística, por cuanto establecen, por lo menos, métodos claros -no así criterios- para delimitar el fenómeno en estudio.

\section{Una propuesta para delimitar la noción de realce}

De la breve exposición anterior se enuncian las siguientes dos críticas generales: a) sobre el fenómeno que nos interesa abordar no existe una definición precisa y coherente con el posterior análisis; en consecuencia, b) se carece, en general, de criterios o procedimientos que permitan delimitar el fenómeno y, por ende, diferenciarlo de otros fenómenos similares o "subfenómenos", de forma de facilitar el diálogo académico intersubjetivo; y, c) las nociones están centradas en productos o actos linguiísticos exclusivamente verbales, lo que no permite su aplicación a textos multimodales. A continuación presentamos una propuesta de definición 
y de criterios y procedimientos para reconocer el realce que buscan solucionar, al menos en parte, estos problemas.

\subsection{El concepto de realce}

El concepto realce se refiere a la ubicación prominente de uno o varios elementos lingüísticos, visuales o sonoros, relacionados entre sí, respecto a otros, llevada a cabo por el emisor y que tiene efectos en el perceptor. En realidad, la definición anterior no se diferencia a primera vista de otras propuestas, por lo cual la explicaremos en detalle.

En primer lugar, el realce constituye una categoría contrastiva. Es una categoría contrastiva, por cuanto lleva a cabo una oposición entre los elementos realzados y una mayoría de elementos-no-realzados implícitos o explícitos. Sin este contraste no hay realce. De esta manera, el realce establece relaciones contrastivas tanto a nivel paradigmático como sintagmático. A nivel paradigmático los elementos realzados se relacionan implícitamente a) con otros de la misma categoría (gramatical, visual, sonora) y, en general, del mismo campo semántico, que potencialmente pudieran ocupar el mismo lugar en el enunciado o en el texto; b) con otras construcciones (en el caso de que sea una construcción compleja como una oración o una frase, imagen de más de un objeto, combinación de colores, más de un sonido) que potencialmente pudieran ocupar el mismo lugar en el enunciado o en el texto; c) con el mismo elemento sin realzar; d) con la posibilidad de omisión de cualquier elemento en ese lugar; e, incluso, e) con la enunciación de un elemento en su lugar (realce por omisión). Para nuestro ejemplo $^{39}$, podemos establecer las siguientes relaciones paradigmáticas (sólo proponemos algunas para el caso de un texto escrito):

Ejemplo: Bolocco pide pisco "original” en Perú.

Opción 1: Bolocco pide pisco falso ${ }^{40}$ en Perú. (relación del tipo a))

Opción 2: Bolocco pide pisco de la casa en Perú. (relación del tipo b))

Opción 3: Bolocco pide pisco original en Perú. (relación del tipo c))

Opción 4: Bolocco pide pisco en Perú. (relación del tipo d))

Las relaciones de los tipos a) y b), en general, no presentan mayor problema y son aceptadas (Gutiérrez 1997: 33). Por su parte, las relaciones c) y d) requieren de aclaraciones. Al señalar que se establece una relación con el mismo elemento sin realzar (relación del tipo c)), presuponemos la posible existencia de un enunciado sin realce, en otras palabras, presuponemos una forma ${ }^{41}$-no-realzada ${ }^{42}$ implícita. Como la relación es paradigmática ${ }^{43}$ debemos enunciar que esa forma-no-realzada ${ }^{44}$ constituye una construcción "de escritorio" (virtual) de los investigadores, quienes recurren a sus conocimientos sobre el uso del código y a otras investigaciones para llevarla a cabo $^{45}$. La relación d) se divide en dos aspectos, por un lado, también se refiere a una posible forma-no-realzada, la que no posee el elemento realzado; y, por otro lado, a la existencia de términos o construcciones que por sí mismas, en determinado contexto, son realce. Así, afirmamos que hay términos y recursos en los códigos que cada vez que se utilicen, generarán realce (por ejemplo, el uso de los superlativos), a los cuales llamaremos elementos realzadores ${ }^{46}$; y otros términos y construcciones que dependerán del contexto (situacional y textual) para producir realce, a los que no asignaremos un nombre englobador.

Un caso diferente se produce en la relación del tipo e), ya que se realza al omitir un elemento. Esto sólo se puede dar en construcciones fosilizadas o muy conocidas en una 
época particular ${ }^{47}$, por cuanto tanto el emisor como el perceptor conocen la construcción y reconocerán la ausencia de un componente esperado, lo que atraerá su atención. Por ejemplo, en Chile una marca de galletas tiene un eslogan que existe hace varios años: "Galletas McKay, más ricas no hay" y fonéticamente 48 /gajetas makkai mas rikas no ai/. Si en alguna campaña publicitaria se dijera /gajetas makkai mas rikas/, llamaría la atención del oyente (conocedor y familiarizado ${ }^{49}$ con el eslogan) la omisión final y, con seguridad, la reconstruiría en su mente, con el consecuente realce del segmento omitido ${ }^{50}$.

A nivel sintagmático, las relaciones se establecen con los otros elementos enunciados, pero que no han sido realzados, los cuales pueden corresponder a todo tipo de categorías gramaticales. En Bolocco pide pisco “original” en Perú la relación se da entre "original” y los otros términos enunciados. De esta manera, se presentan varios elementos-no-realzados, pero que no son construcciones "de escritorio" (como sí lo son las formas-no-realzadas), sino que aparecen en el mismo texto. Estas relaciones contrastivas son semánticas y, además, jerárquicas perceptivas dentro de la estructura a la que pertenecen. Son semánticas, por cuanto el realce facilitaría (hipótesis) la generación de significados asociados del elemento realzado y la reduciría de los elementos-no-realzados. Por su parte, las relaciones contrastivas jerárquicas perceptivas se refieren a la manifestación física del objeto (enunciado, imagen, sonoridad), en la que el realce posiciona en un lugar prominente perceptivamente a los elementos realzados frente a los elementos-no-realzados. En nuestro ejemplo se da por el uso de comillas.

En segundo lugar, a partir de lo anterior, cuando enunciamos "ubicación prominente" en nuestra definición, se debe entender, primero, como ubicación semántica prominente y, luego, como ubicación perceptiva prominente. Ambas ubicaciones prominentes no son excluyentes entre sí ni se dan, necesariamente, a la vez. La ubicación semántica prominente se refiere a los grados extremos $^{51}$ (o grado superior) dentro de una escala de fuerza (White 1999) en un contexto particular. Esa gradación puede generarse a partir, por un lado, de que el significado asociado con el elemento realzado o realzador pertenece a una escala dentro del sistema del código (por ejemplo, superlativos en el lenguaje verbal) o, por otro lado, de una gradación efectuada por el elemento realzador semántico (por su significado) en el uso, en el texto (por ejemplo, la estructura sintáctica "no sólo (solamente)... sino (que)..."). De esta manera, la prominencia semántica no corresponde necesariamente a lo más importante informativamente (como sucedería con el foco), sino al grado extremo de una gradación. Esto deja abierta la posibilidad de que el elemento realzado coincida con lo más importante informativamente, en especial, cuando la gradación se da en el texto y no previamente en el sistema. La ubicación semántica prominente delimita la (re)construcción de la forma-no-realzada y la identificación de los elementos-no-realzados, ya que en ambos casos no deben corresponder a un grado superior de la escala (a la cual pertenece el elemento realzado); de hecho, la forma-no-realzada no debe expresar esa gradación. Así, no cualquier posible relación sintagmática es relevante para el análisis del realce semántico, sólo aquellas que pertenezcan a la escala del elemento realzado o realzador.

La ubicación perceptiva prominente es un aspecto dejado de lado en otras definiciones, debido a que se han centrado en el fenómeno lingüístico, olvidando que todo uso de cualquier lenguaje (o combinación de ellos) tiene su manifestación física que también hay que tener presente. Ahora bien, la percepción la entendemos como el proceso de construcción de representaciones a partir de estímulos externos (ondas acústicas, luminosas, etc.). Este proceso no es exhaustivo, ya que no percibimos todas las ondas que nos rodean, debido a 
limitaciones de nuestros órganos sensoriales (por ejemplo, hay ondas acústicas que no somos capaces de percibir) y a que construimos basándonos en nuestras representaciones previas ${ }^{52}$, y es selectivo, fijamos nuestra atención (a partir de instinto de supervivencia, conocimientos previos, intereses personales) en algunas cosas y no en todos los impulsos que nos pueden llegar del ambiente (Roth 1997: 248). Por lo anterior, en el proceso de percepción (como en el de interpretación) realizamos predicciones sobre lo que estamos percibiendo (completamos la información de lo que no percibimos por deficiencia de nuestros órganos o del impulso o, incluso, "corregimos" lo que percibimos, porque no se ajusta a nuestro conocimiento previo ${ }^{53}$ y, en consecuencia, a lo que esperábamos) y sobre lo que vamos a percibir (Frith 2008: 161). De esta manera, la prominencia perceptiva ${ }^{54}$ se genera de dos formas: primero, al facilitar la percepción de algo, es decir, el elemento realzado es más fácil de percibir, debido a la calidad del impulso ${ }^{55}$, o sea, que nuestros órganos sensoriales puedan identificar con facilidad los impulsos (por ejemplo, en la oscuridad no vemos las mismas cosas que en lugares luminosos), y a que nuestro conocimiento previo (de los códigos, del tipo de texto, de la situación) nos permite reconocer que algo ha sido realzado (por ejemplo, el uso de cursivas en un texto verbal escrito ${ }^{56}$ ); y, segundo, al romper con lo que se espera que se manifieste (perciba) en un lugar determinado, en otras palabras, al romper con una predicción casi siempre acertada ${ }^{57}$ (por ejemplo, cuando un objeto aparece con color dentro de una película en blanco y negro, o el caso del slogan de las galletas Mckay), donde el elemento realzado es lo no esperado perceptivamente. Para esto último, se requiere que ese rompimiento pueda ser percibido y no sea "corregido" en el proceso de percepción. A partir de esto, se delimita la (re)construcción de la forma-no-realzada en un realce "perceptivo", por cuanto la forma-no-realzada corresponde a lo que en la mayoría de las veces aparece en la ubicación donde se encuentra el elemento realzado. Por ende, esa forma-no-realzada tiene que ser aceptada (dentro de un grupo social) como lo esperado en esa ubicación ${ }^{58}$. De esta manera, cualquier construcción especulativa o no aceptada de una (posible) forma-no-realzada no se considera como la formano-realzada, lo cual significa que el investigador deberá construir otra forma-no-realzada o que no se está frente a un realce (por lo menos, de este tipo).

En tercer lugar, la principal función del realce es destacar un(os) elemento(s) sobre otros, sin embargo, no hay una especificidad con relación al aspecto realzado. Así, en algunos casos puede destacar una cualidad (característica) de un objeto, a un objeto o persona, un punto de vista, una idea, una actitud o una sensación (no explícita), los cuales no son necesariamente excluyentes entre sí (por ejemplo, se puede realzar una cualidad y un objeto: en "el peor Maradona llegó al equipo" se realza a la persona Maradona y a una cualidad de ésta, peor).

En cuarto lugar, ante el aumento de la producción de textos multimodales (multimediales, multidimensionales) y, en consecuencia, de los estudios sobre éstos, no se debe reducir el fenómeno del realce a un código en particular ni a un código aislado. Por este motivo, se excluye de la definición cualquier referencia que reduzca el ámbito de aplicación del término a un código o a relaciones dentro del mismo código. De esta manera, las relaciones paradigmáticas y sintagmáticas también hay que conceptualizarlas de una forma amplia, es decir, que no se enmarcan en cada código, sino que pueden darse entre elementos de distintos códigos (por ejemplo, entre lo verbal, lo visual ${ }^{59}$ y lo sonoro). Por lo tanto, las relaciones semánticas (ubicación prominente) se tienen que entender como relaciones entre distintos tipos de representaciones ${ }^{60}$ (verbales, visuales, acústicas). No obstante, creemos que el problema en los textos multimodales radica en que entre los códigos utilizados se establecen diferentes 
relaciones (explicación, nuevos elementos, contradicción, ejemplificación de lo que se presenta con otro código), debido a que los códigos avanzan de formas paralelas: independiente, cuando cada código manifiesta contenidos que no tienen relación entre si $\mathbf{1}^{61}$ (muy raros casos); complementaria coordinada, cuando cada código en un mismo nivel de jerarquía aporta contenidos que complementan el todo; complementaria subordinada, cuando el contenido manifestado por un código se encuentra al servicio del contenido de otro código. Estas relaciones pueden ser constantes (durante todo un texto) o variables (en diferentes momentos del texto). Mi hipótesis es: esto repercutiría en el realce, por cuanto se puede dar de forma integral $^{62}$ (un realce que afecta al texto como un todo) y de forma paralela ${ }^{63}$ (cuando se presenta más de un realce al mismo tiempo, es decir, en diferentes códigos).

En quinto lugar, en la definición no se hace referencia a la conciencia o la intencionalidad del emisor, porque se busca liberar al hablante de la responsabilidad sobre el porqué y para qué del realce. No interesa si un emisor deseaba o no realzar, simplemente lo hizo, es decir, existe realce independientemente de la conciencia del hablante. Así, esta definición intenta dejar de lado analíticamente el presupuesto de que se realza conscientemente con una finalidad determinada, ya que ese presupuesto limita el acercamiento al fenómeno por parte de los analistas. Éstos, en muchas ocasiones, se enfrentan (nos enfrentamos) a situaciones donde no es claro (ni por el texto ni por el contexto) si el hablante fue consciente o no de haber efectuado realce $y$, al no poder contactar al hablante para consultarle, le quedan sólo dos opciones: aceptarlo o rechazarlo como realce. Asimismo, no estamos de acuerdo con asignarle una finalidad (u objetivo) al realce, ya que también limita el acercamiento, por cuanto no permite identificar diferentes funciones en su uso particular o a nivel textual. Cabe destacar que con esto también se deja abierta la posibilidad de que los analistas logren identificar que el hablante realzó conscientemente ${ }^{64}$ y con una finalidad específica. De esta manera, no conceptualizamos al realce como una estrategia, por cuanto no consiste, necesariamente, en un plan de prácticas intencionales con un objetivo particular ${ }^{65}$. Esto no anula la posibilidad de que el realce en algunos usos constituya una estrategia determinada ${ }^{66}$.

En sexto lugar, el realce no debe ser un recurso recurrente dentro de un texto. Si fuera recurrente, el perceptor se enfrentaría a tantos elementos realzados que no tendrían ninguna particularidad y no se podría hablar de prominencia, ya que esa ubicación destacada se anularía o disminuiría su fuerza (su impacto) perceptiva ${ }^{67}$. No somos capaces de establecer una relación estadística entre el uso del realce y la cantidad de elementos-no-realzados (palabras, objetos, sonidos, enunciados), por lo cual queda, por el momento, a criterio del investigador ${ }^{68}$.

Finalmente, como ya señalaron Albelda ${ }^{69}$ (2005) y Kiesler (1989), el realce produce efectos en el texto, en el hablante, en el oyente y en la interacción. No obstante, estos efectos son sólo potenciales, por cuanto dependen de factores externos al realce (por ejemplo, que el oyente se percate del realce, que el realce sea consciente, que el realce sea tomado en cuenta, entre otros) para que se concreten. Por este motivo, no consideramos posible entregar una lista cerrada de efectos del realce, sino que nos referiremos a algunos posibles. A nivel textual el realce ${ }^{70}$, además de establecer relaciones paradigmáticas y sintagmáticas, puede reforzar las fuerzas locutiva e ilocutiva del acto de habla en el que se encuentra (Briz 1998: 111; Albelda 2005). A nivel del hablante es capaz de manifestar su expresividad e intensificarla (Vigara 1992), con lo que le otorgaría mayor fuerza a la posición (punto de vista) del hablante (Albelda 2005). En el oyente el realce podría producir: en primer lugar, mayor atención sobre el elemento realzado por parte del oyente, lo que conllevaría un mejor almacenaje y retención en la memoria, por ende, 
ese elemento sería más fácil de recordar; asimismo, al concentrar el oyente mayor atención en el elemento realzado, esto puede generar sensaciones ${ }^{71}$ fuertes al respecto, siempre y cuando, ese elemento tenga cargas afectivas claras (intensas) para el oyente; por último, puede guiar las inferencias que realice el oyente y, por lo tanto, su interpretación y comprensión de lo percibido. En cuanto a la interacción, el realce permitiría regular la negociación de acuerdos o desacuerdos (Albelda 2005) y, además, ayudaría a delimitar el tipo de interacción (se permite realzar tal o cual elemento y mediante determinados recursos).

A partir de los puntos tratados anteriormente, precisamos de la siguiente manera nuestra definición de realce: realce es la ubicación prominente perceptiva o semántica de uno o varios elementos relacionados entre sí en oposición a una mayoría no realzada, tanto a nivel sintagmático como paradigmático, la cual ejecuta (de manera consciente o inconsciente) el emisor y puede tener efectos en el texto, el emisor, el perceptor y la interacción. Con esta definición amplia no limitamos su utilización a un tipo particular de texto (por ejemplo, conversación) o registro ${ }^{72}$ (coloquial), ni a un código particular (verbal, audiovisual, de la imagen fija), ni a una determinada perspectiva lingüística (pragmática, gramática). Además, su identificación depende, principalmente, del texto y, en menor medida, del contexto (esencialmente, situación y cotexto), pero no de los participantes ${ }^{73}$ de la interacción, sobre quienes, eso sí, puede producir efectos. Esto no restringe su tratamiento a una perspectiva, sino, por el contrario, abre la posibilidad de que se aborde el realce desde distintas disciplinas y puntos de vista, acercamientos que permitirán comprender mejor el fenómeno y establecer relaciones entre los recursos para realzar y los contextos de uso (género textual, tipo de texto, registro, modo).

\subsection{Sobre el procedimiento para reconocer el realce}

Si bien hemos dado una definición del realce, no hemos señalado, hasta el momento, el procedimiento para identificarlo. Consideramos que para lograr su identificación siempre se debe reconstruir la forma-no-realzada o realzada de otra manera e identificar los elementosno-realzados. Que el realce sea un fenómeno contrastivo significa que la existencia de esa forma-no-realzada o esos elementos-no-realzados (con los cuales se da el contraste) constituye un requisito esencial. Así, cada vez que se desee abordar el realce, se tendrá necesariamente que reconstruir e identificar esas formas-no-realzadas y elementos-no-realzados. Podemos enunciar: sin una forma-no-realzada o elementos-no-realzados no existe realce.

Ahora bien, al mismo tiempo que se realiza lo anterior, se debe identificar el recurso utilizado para realzar. A continuación expondremos algunos de esos recursos.

\subsubsection{Recursos para realzar}

Dentro de los recursos para realzar se distinguen entre los elementos realzadores y aquellos recursos que producen realce debido al contexto (situación, cotexto, tipo de texto, registro) en que son utilizados. Los elementos realzadores corresponden a aquellos recursos de los códigos (por separados y en combinación) que "cada vez"74 que son utilizados producen realce. Estos elementos realzadores tienen esa capacidad debido al proceso dinámico de construcción de los códigos sociales. Es decir, gracias a un proceso histórico de creación de medidas comunicativas han asignado a esta función esa capacidad, pero por el mismo proceso pueden perderla. De esta manera, cuando afirmamos que los elementos realzadores siempre 
que se usen producen realce, se debe entender dentro de un contexto socio-histórico particular. Por otra parte, hay un sinnúmero de otros recursos que sólo en determinados contextos de uso (situación y cotexto) son capaces de generar realce. A continuación se explican los elementos realzadores ${ }^{75}$.

\subsubsection{Elementos realzadores}

Como ya hemos señalado, se puede distinguir entre una prominencia semántica y otra perceptiva, las cuales no son excluyentes entre sí, por lo tanto, pueden presentarse juntas. Esta distinción también se la aplicamos a los elementos realzadores. De esta manera, hay dos tipos de elementos realzadores: semánticos y perceptivos.

\subsection{Elementos realzadores semánticos}

Este tipo de recursos produce realce gracias a su uso y a la red de asociaciones que genera, es decir, su contenido semántico es el responsable de la prominencia sobre el resto de elementos. Ahora bien, hay recursos que producen y constituyen ellos mismos el elemento realzado y otros que sirven de instrumento para realzar a otro elemento, o sea, sólo producen realce y no constituyen el elemento realzado. A partir de los análisis efectuados, afirmamos que ambos tipos de recursos (los que producen realce y son el elemento realzado y los que sólo producen) se caracterizan por constituir el grado extremo de una escala evaluativa o producir una gradación explícita. Sin embargo, se diferencian en que los segundos (los que sólo producen realce) determinan o modifican a otros elementos.

Una escala es un continuum compuesto por elementos graduales que se ubican en distintos lugares desde una base a un extremo (aquellos que se ubican en los extremos producirían realce). Entre los elementos graduales hay una relación contrastiva, ya que se oponen a los otros elementos, de hecho, necesitan de su existencia para ser miembros de una escala. Al darse esta relación contrastiva, para que haya realce se requiere de formas-norealzadas dentro de esa escala, las cuales pueden considerarse el elemento cero del continuum ${ }^{76}$ (como toda forma-no-realzada, ese elemento cero constituye una construcción virtual con fines científicos). Sin embargo, si se toma en cuenta el uso del lenguaje, observamos que algunos componentes de las escalas y su ubicación pueden variar. Esto dificulta la identificación de los conformación de cada escala. Para solucionar este problema se puede seguir lo normativo o los usos en contextos particulares.

Dentro de estos elementos realzadores semánticos hemos identificado: adjetivos calificativos en función atributo, adjetivos comparativos en función sustantiva, gradación de adjetivos calificativos mediante el uso del adverbio más, las enumeraciones, la estructura sintáctica "no sólo (solamente).... sino también...", entre otros. Cabe señalar que las listas de recursos que se presentan se originan sólo de un primer acercamiento exploratorio al realce.

\subsection{Elementos realzadores perceptivos}

Este tipo de recurso se caracteriza por existir gracias a la oposición sintagmática, es decir, al contraste explícito (perceptible) entre elemento realzado y elementos-no-realzados. De esta manera, los elementos-no-realzados evidentemente también son percibidos, sin embargo, no ocupan esa posición prominente en el sintagma. 
Como ya se señaló, la percepción es un proceso en el cual participan conocimientos (representaciones y asociaciones) previos de las personas, por lo tanto, las diferencias sociales, culturales, pueden conllevar distintas percepciones, además de las diferencias perceptivas entre códigos (por ejemplo, entre el castellano y las lenguas tonales). Por este motivo, no se puede universalizar $^{77}$ los elementos realzadores perceptivos, sino tan sólo enmarcarlos en una cultura o un código particular.

Algunos de los elementos realzadores perceptivos que hemos identificado en textos multimodales (audiovisuales) en lengua española son: pronunciación enfática, repetición, ubicación en el centro del cuadro, movimiento de la cámara, movimiento del zoom, entre otros.

\section{Conclusiones}

En el presente texto hemos presentado una propuesta para la delimitación del concepto de realce y para su identificación basada en una conceptualización amplia del fenómeno. Esta conceptualización permite abordarlo, dejando de lado presupuestos previos, en especial, como aquellos que responsabilicen al hablante de la producción del realce conscientemente y con una finalidad clara o que se centren en un código en específico (el verbal). Nuestra postura se fundamenta en que como hablantes en muchas ocasiones realzamos (ubicamos en una posición prominente semántica y, principalmente, perceptiva) sin una intención ${ }^{78}$ específica de hacerlo ni tampoco tenemos siempre claridad sobre los posibles efectos del realce y, asimismo, existen características contextuales que nos obligan a realzar (por ejemplo, géneros discursivos particulares, situaciones).

No obstante, esto es una propuesta, por lo cual se deben realizar más investigaciones con el fin de confirmar, precisar o corregir las definiciones, explicaciones e hipótesis. Consideramos que las tareas futuras se dividen en tres áreas de investigación: por un lado, la descripción de los recursos para realzar en distintas realizaciones lingüísticas, con el objetivo de identificar los recursos contextuales y los elementos realzadores (semánticos o perceptivos), observar su relación con tipos de texto, géneros discursivos o situaciones. Por otro lado, la relación entre realce y otras nociones cercanas como focalidad, foco, énfasis, intensificación, atenuación, relieve o saliencia, para delimitar si el realce constituiría un concepto que abarcaría todas esos fenómenos o, más bien, sólo alguno de ellos (tenemos dudas de que, por ejemplo, todo recurso para producir foco o focalización corresponda a un realce); y, por último, el análisis de los efectos que podría tener el realce y los diferentes recursos para realzar a nivel personal, textual, interactivo, cognitivo y del proceso de comprensión, con el fin de comparar las hipótesis de que el realce facilitaría la generación de significados asociados del elemento realzado y disminuiría la de los elementos-no-realzados, lo cual podría influir en la comprensión de un texto, además de la construcción, "almacenamiento" y recuperación de representaciones (mentales); y confirmar la hipótesis de que el realce no debe ser recurrente dentro de un texto, ya que reduciría su particularidad y, por ende, sus posibles efectos.

\section{Notas}

1. A pesar de ser un término poco preciso, utilizamos elemento, debido a que puede variar el tipo de código, los objetos y la naturaleza (semántico, expresivo, emotivo, acción) de lo realzado.

2. Extraída el 29-04-08, de http://cl.msn.com/

3. Cecilia Bolocco es una ex Miss Universo de Chile que posteriormente ha animado diferentes programas de televisión y, asimismo, se casó con el ex presidente de Argentina, Carlos Menem. 
4. Aquí no se discute la función de la utilización de las comillas (“"), por lo cual no entraremos en si el uso es de distanciamiento, ironía o cualquier otro.

5. La disputa se puede resumir en que el pisco (un aguardiente de uvas) tradicionalmente es una bebida alcohólica relacionada con Perú, aunque en Chile también se ha consumido, sin embargo, empresarios chilenos patentaron el producto pisco internacionalmente, lo que significó que los productores peruanos no pudieran exportar este aguardiente con ese nombre.

6. Los recursos sonoros se diferencian de los prosódicos en que se refieren a sonidos no relacionados con el lenguaje verbal, como música, ruido ambiente, ruido provocado por objetos u animales.

7. Cabe señalar que consideramos que una buena definición de un fenómeno lingüístico debe ser de tal claridad que permita o facilite la identificación del fenómeno (por supuesto, mediante la aplicación de un método de análisis). Por este motivo, en esta revisión se hace énfasis en la posibilidad de identificar los fenómenos a partir de las definiciones.

8. Las propuestas expuestas en este texto no son las únicas, pero sí consideramos que son representativas dentro de sus perspectivas teóricas. Además, cabe señalar que se hace referencia a textos particulares y no, necesariamente, a autores, por cuanto no hemos tenido acceso a todos los textos de ellos sobre el tema.

9. $\quad$ En este texto se hace una revisión del concepto de foco.

10. Nuestra traducción de los términos alemanes Hervorhebung, Alternative y Ausgesagtes respectivamente.

11. Afirmamos que "carece de una comprobación empírica", por cuanto no hay pruebas de que cada persona que haya "focalizado" un elemento haya sido consciente de haberlo hecho.

12. Miguel Metzeltin utiliza los términos realce y énfasis, sin embargo, consideramos que prefiere el término énfasis, ya que distingue entre "énfasis absoluto" y "énfasis contrastivo" (1990: 169-174).

13. Creemos que en Gutiérrez (1997), al no darse criterios para la delimitación del foco y presentarse un número cerrado de recursos, se identifica como foco exclusivamente los casos en que se utilicen esos recursos lingüísticos. Esto sería coherente y, por ende, aceptable si se hubiese otorgado una definición más cerrada del fenómeno, es decir, no incluir al hablante.

14. El texto de Vigara (1992) aborda el español coloquial.

15. Esto no sería así, si se efectuaran experimentos con muchos hablantes para comprobar si la intuición del investigador es la correcta.

16. Dentro de nuestro concepto de texto se pueden incluir conversaciones, textos escritos, productos audiovisuales o visuales, entre otros.

17. Nos referimos a las características generales del intercambio, ya que los analistas no tienen acceso en todas las ocasiones a detalles (por ejemplo, intención del hablante, efectos sobre el oyente).

18. De hecho, Albelda (2005) la incluye entre los autores que han analizado la intensificación. Además, en el mismo texto de Vigara aparecen fragmentos que fomentan esta confusión: "En su afán enfatizador, el hablante llega continuamente, de forma inconsciente, al incumplimiento de las restricciones en la aparición de marcas intensificadoras" (1992: 152). ¿A qué se refiere con "enfatizador" y "marcas intensificadoras"?

19. En general, esta autora sigue las propuestas de Antonio Briz (1995, 1998).

20. En su trabajo doctoral, Marta Albelda (2005) realiza un análisis profundo de diferentes términos y definiciones lingüísticas que abordan fenómenos cercanos o similares a la intensificación. Su acercamiento se diferencia del nuestro, por cuanto a ella le interesa la intensificación y no el realce, por lo cual, parte de otro punto de vista. Sin embargo, coincidimos en varias críticas: ambigüedad en las definiciones, trabajos intuitivos, falta de criterios para una delimitación clara de los fenómenos. A partir de esa revisión crítica establece su definición de la intensificación.

21. Según Albelda, "constituyen un todo en el discurso real" (2005: 184).

22. Suponemos que por "consciente" la autora entiende que los hablantes tenían la finalidad de realizar la intensificación.

23. Ulrich Klein (1992) aborda la relación entre acento y foco en el alemán, como relación entre "Hervorhebung" de contenido y gramatical respectivamente. Lamentablemente, no entrega ninguna definición de Hervorhebung. 
24. Jürgen Kunze (1991) analiza la relación entre casos (Kasus), roles semánticos, relaciones semánticas y el énfasis semántico (semantische Emphase) en el alemán. Esto lo lleva a cabo desde la semántica y la sintaxis, con especial influencia de la gramática generativa y la lingüística cognitiva. El énfasis semántico corresponde a un objeto de estudio específico (contenido proposicional) y se aleja del fenómeno que nos interesa. Esto no excluye la posibilidad que este énfasis semántico pueda incluirse dentro del estudio del realce, por supuesto, desde una perspectiva teórica particular.

25. Llama la atención que la mayoría de los trabajos referidos son anteriores a 1970. No hemos recurrido a estos trabajos, porque nos parecen acertados los comentarios y críticas que se les hacen.

26. Utilizaremos el término en alemán para evitar confusiones con la terminología lingüística en castellano.

27. Traducción nuestra del original en alemán: "Hervorhebung ist jede während eines sprachlichen Produktionsvorganges sich vollziehende Umänderung - welche sich auf der lokutionären Ebene der Äusserung niederschlägt -, die bewusst oder unbewusst teleologisch orientiert eine Intensivierung der erstrebenten perlokutionären Wirkung der Äusserung zum Ziele hat. Durch eine dergestalt veränderte Äusserung wird ein Mehr an Information vermittelt” (Kiesler 1989: 82).

28. Kiesler habla de término relacional o de relación (Beziehungsbegriff), la cual es contrastiva.

29. Se debe tener presente que al decir "otro enunciado similar", nos referimos a enunciados casi idénticos, en los cuales se eliminaría el realce. Así, por ejemplo, en el caso del enunciado Bolocco pide pisco "original" en Perú, ese "otro enunciado similar" sería Bolocco pide pisco original en Perú.

30. Kiesler conceptualiza a la intensificación (Verstärkung) y a la atenuación (Abschwächung) como dos funciones de la Hervorhebung: respectivamente, como el aumento y como la disminución de la intensidad del efecto deseado. "Verstärkung ist jede durch Umänderung auf der lokutionären Ebene einer Äusserung bewirkte Vermehrung der Intensität der erstrebten perlokutionären Wirkung dieser Äusserung. [...] Abschwächung ist jede durch Umänderung auf der lokutionären Ebene einer Äusserung bewirkte Verminderung der Intensität der erstebten perlokutionären Wirkung dieser Äusserung" (1989: 83).

31. Entendido como posicionar una información como más relevante que otra y como aporte de más información de la que se hubiera otorgado en caso de que no hubiera un realce (Hervorhebung).

32. Esos efectos son deseados por el hablante, pero no necesariamente se producen en el interlocutor, son tan sólo potenciales.

33. Lo semántico se refiere a la mayor información del enunciado.

34. Nuestra traducción del ejemplo en portugués: "E eu hoje creio que era bastante assim" (Kiesler 1989: 101). Las cursivas son nuestras.

35. Pondremos en cursivas la posibilidad normal de enunciado.

36. Nuestra traducción del ejemplo en portugués: "E a tua burra é um excelente animal. Foi negocio" (Kiesler 1989: 102).

37. Del portugués "De ideologias estamos todos fartos" (Kiesler 1989: 102). Las cursivas son nuestras.

38. Del portugués "Seriam umas dez horas quando cheguei à vila" (Kiesler 1989: 103). Las cursivas son nuestras.

39. El contraste e) no se puede explicar a partir de nuestro ejemplo. Más adelante lo abordamos por separado.

40. Las negritas señalan el elemento con el cual se establecería la relación paradigmática (Nótese que con las negritas se realza ese elemento).

41. Utilizamos el término forma para referirnos a un componente lingüístico implícito en el marco de esta propuesta sobre el realce, mientras que preferimos elemento para aquellos explícitos y, por ende, perceptibles. Así, cuando se hable de forma-no-realzada, se debe entender algo implícito (reconstruido por el analista).

42. En términos de Vigara (1992) y de Kiesler (1989) correspondería a la "forma no marcada" y a la "posibilidad normal" respectivamente.

43. Más adelante veremos que también se dan relaciones con elementos-no-realzados a nivel sintagmático.

44. En unos párrafos más adelante desarrollaremos las ubicaciones semántica y perceptiva prominentes, a partir de las cuales se delimita esa forma-no-realzada. 
45. Al recurrir a otras investigaciones, el analista facilita el diálogo intersubjetivo y reduce su protagonismo (su subjetividad) en la construcción de la forma-no-realzada. Sin embargo, en la mayoría de los casos no existen esas investigaciones de respaldo respecto al español.

46. Más adelante se aborda con mayor profundidad los elementos realzadores.

47. Nos referimos a construcciones que un momento histórico particular (campaña electoral, evento deportivo, campañas publicitarias) son muy conocidas.

48. Según la simbología del Alfabeto Fonético Internacional (AFI).

49. Por familiarizado entendemos cuando una construcción, además de ser conocida, es parte de la vida cotidiana de una persona (la escucha a diario, la reproduce de vez en cuando).

50. En este ejemplo consideramos que el realce de ese elemento omitido conlleva, de alguna manera, un realce de toda la estructura a la que pertenece (todo el eslogan).

51. En el uso del lenguaje las escalas (sus miembros y su ubicación en la gradación) pueden variar, por lo cual es más acertado hablar de grados extremos, así no se cierra la posibilidad de que en otro contexto de uso sea otro elemento el grado extremo de la escala.

52. En el proceso de percepción también hay una fase precognitiva (sin utilización evidente de representaciones previas), en la cual se construyen representaciones básicas de las ondas recibidas (contrastes, movimiento, colores, claridad) (Roth 1997: 258), sin embargo, en esta fase todavía no se puede reconocer lo percibido (¿hacia dónde se mueve?, ¿qué colores vemos?, ¿qué vemos?). En otras palabras, cuando hablamos de percepción, nos referimos al momento en que las personas reconocen que han percibido algo (aunque no sepan qué cosa es, pero pueden diferenciarla de cosas ya conocidas).

53. Por ejemplo, el ser humano es "incapaz" de percibir una cara cóncava, ya que su conocimiento previo le "dice" que las caras son convexas (Frith 2008: 164-165).

54. Presuponemos que la atención está dirigida al texto.

55. Dentro de la calidad de un impulso incluimos la modalidad de la onda (luminosa, táctil, acústica), la submodalidad (por ejemplo, color, movimiento, claridad), la intensidad (por ejemplo, volumen, claro, oscuro) y la duración (Roth 1997: 108-109).

56. Por supuesto, se presupone que el texto no está completamente escrito con cursivas.

57. "Casi siempre acertada" se refiere a aquellas predicciones que se realizan a partir de nuestros conocimientos previos y que en una mayoría casi absoluta (digamos en más del 90\%) concuerdan con lo que percibimos (por ejemplo, si vemos que se abre la puerta de un avión comercial, esperamos que salgan seres humanos y desciendan por la escalera, así si de repente vemos aparecer un animal, se romperá la predicción que habíamos realizado y se produciría un tipo de "shock perceptivo").

58. Dentro de esta forma de generarse el realce podrían incluirse las formas sintácticas que se alejan del uso mayoritario (normal) dentro de un contexto determinado. Eso sí, deberían ser rompimientos claros de lo esperado, no construcciones facultativas.

59. Un ejemplo que se nos ocurre es el siguiente: En un programa de televisión nos presentan un vídeo resumen del discurso realizado por un político frente a una multitud. El texto audiovisual muestra al político hablando frente a la gente (sin nada fuera de lo "normal") y se escucha sin problema lo que está diciendo, sin embargo, de repente, por medio de un efecto visual, le comienza a crecer la nariz al político (a lo Pinocho). Aquí nos encontramos frente a un realce, en el cual se realza una valoración (evaluación, opinión) sobre el político, mediante un recurso visual. Hasta antes del crecimiento de la nariz el código verbal era guía del texto (manifestaba mayor cantidad de contenido relevante), no obstante, fue mediante el visual que se generó el realce.

60. Hemos utilizado ejemplos del código verbal para explicar las relaciones paradigmáticas, ya que el estudio de este tipo de relaciones entre diferentes códigos se aleja de nuestros textos analizados.

61. En ocasiones parece que cada código se presenta independientemente, sin embargo, al observar detenidamente o al analizar el texto como un todo, nos percatamos que sí existe relación entre ellos.

62. Como en el ejemplo dado del discurso público de un político.

63. En una noticia mientras se realza verbalmente el número de puñaladas dadas, se realza visualmente el llanto de una mujer. 
64. Además, se debe tener presente la diferencia entre efectuar un acto intencionalmente (por ejemplo, pronunciar una palabra) y efectuar realce intencionalmente (relación contrastiva).

65. Seguimos la definición de Reisigl y Wodak: "By 'strategy' we generally mean a more or less accurate and more or less intentional plan of practices (including discursive practices) adopted to achieve a particular social, political, psychological or linguistic aim" (2001: 44).

66. No se puede considerar ni conceptualizar el realce, a priori, como una estrategia, por cuanto se requiere de un estudio detallado de sus usos y contextos de usos para clasificarlo como tal. A partir de los resultados de nuestra investigación, no podemos generalizar el uso del realce. Además, no deseamos circunscribir el término realce a una perspectiva teórica particular (por ejemplo, pragmática o análisis del discurso), por lo cual no partimos, para su definición y explicación, de presupuestos como tareas, metas, planes o tácticas discursivas.

67. Por ejemplo, un texto visual en el que se observaran muchos objetos grandes y con colores llamativos. En este caso esos objetos llamativos (realzados) perderían su capacidad de destacar frente a los otros (su realce), de hecho, se habla cotidianamente de un texto (dibujo, pintura, fotografía) "recargado".

68. Este es uno de los puntos que se deben profundizar para comprender mejor el realce.

69. Pareciera que la intensificación y, a su vez, la atenuación constituyen recursos para realzar.

70. No incluimos entre los efectos que el realce destaca un elemento de un enunciado, por cuanto es su función esencial, lo que lo hace ser realce.

71. Se debe recordar que no hay percepción sin sensaciones.

72. Creemos que según el género textual, el tipo de texto y el registro varían los recursos para realzar y los elementos que se realzan.

73. Como se observa, se excluye de la situación a los participantes de la interacción.

74. En realidad, debemos señalar que los elementos realzadores casi siempre -y no cada vez- que son utilizados producen realce, ya que no hemos tenido acceso a todos los posibles usos de estos recursos, por lo cual no podemos asegurar que siempre produzcan realce.

75. En este artículo sólo explicamos los aspectos teóricos del realce y de los recursos para realzar.

76. El elemento cero del continuum puede corresponder a aquel elemento ubicado en el inicio o en el medio de una escala, lo cual depende de la naturaleza de la escala (si tiene dos extremos, es decir, dos proyecciones, o sólo uno).

77. Por supuesto, habrá recursos para realzar compartidos entre lenguas y culturas.

78. Por intención entendemos una disposición mental hacia la realización de un acto. Esto se diferencia de la finalidad del acto, ya que la finalidad dependería del acto (y de su realización, es decir, correspondería al objetivo buscado al realizar el acto), en cambio, el acto dependería de la intencionalidad (es decir, de la disposición mental del actor para realizar el acto, lo cual es independiente de la finalidad del acto).

\section{Bibliografía}

Albelda Marco, Marta. 2005. La intensificación en el español coloquial. Valencia: Servei. Recuperado de: http://www.tesisenxarxa.net/TESIS_UV/AVAILABLE/TDX-0701105125232//albelda.pdf. Consulta: 20 de noviembre de 2008.

Alcaraz Varó, Enrique y María Antonia Martínez Linares. 2004. Diccionario de lingüística moderna. Barcelona: Ariel.

Austin, John L. 1962. How to do Things with Words. Cambridge: Harvard University Press.

Briz, Antonio. 1995. "La atenuación en la conversación coloquial. Una categoría pragmática". En: El español coloquial. Actas del I simposio sobre análisis del discurso oral. Almería, 23-25 de noviembre de 1994. Luis Cortés Rodríguez (Ed.). Almería: Universidad de Almería. 101-122. 
1998. El español coloquial en la conversación. Esbozo de pragmagramática. Barcelona: Ariel.

Dimroth, Christine. 2004. Fokuspartikeln und Informationsgliederung im Deutschen. Studien zur deutschen Grammatik 69. Tübingen: Stauffenburg.

Face, Timothy L. 2002. International Marking of Contrastive Focus in Madrid Spanish. München: Lincom Europa.

Frith, Chris. 2008. Descubriendo el poder de la mente. Cómo el cerebro crea nuestro mundo mental. Barcelona: Ariel.

Gutiérrez Ordóñez, Salvador. 1997. Temas, remas, focos, tópicos y comentarios. Cuadernos de Lengua Española. Vol. 46. Madrid: Arco Libros.

Kiesler, Reinhard. 1989. Sprachliche Mittel der Hervorhebung in der modernen portugiesischen Umgangssprache. Heidelberg: Carl Winter Universitätsverlag.

Klein, Ulrich F. G. 1992. Fokus und Akzent. Bemerkungen zum Verhältnis von inhaltlicher und grammatischer Hervorhebung. Hürth-Efferen: Gabel.

Kunze, Jürgen. 1991. Kasusrelationen und semantische Emphase. Berlin: Akademie.

Metzeltin, Miguel. 1990. Semántica, pragmática y sintaxis del español. Wilhelmsfeld: Egert.

Pinuer, Claudio. 2009. "La dimensión de focalidad: conceptualización, instanciación y taxonomía". Revista Signos. 42 (69): 83-106.

Roth, Gerhard. 1997. Das Gehirn und seine Wirklichkeit. Neurobiologie und ihre philosophischen Konsequenzen. Frankfurt am Main: Suhrkamp.

Vergara Heidke, Adrián. 2008. "Análisis crítico del sensacionalismo: la construcción mediática de la criminalidad en la televisión costarricense". Iberoamericana. 8 (32): 99-117.

Vigara Tauste, Ana María. 1992. Morfosintaxis del español coloquial: esbozo estilístico. Madrid: Gredos.

White, Peter R. R. 1999. "Un recorrido por la teoría de la valoración”. Recuperado de: www. grammatics.com/valoracion/ 
